\title{
Lagrangian, Hamiltonian, and Energy-based Control for Space Tethered System
}

\author{
Junjie Kang and Zheng H. Zhu \\ York University \\ Toronto, Canada \\ kangjunjie1989@gmail.com; gzhu@yorku.ca
}

\begin{abstract}
A typical and useful way to derive the dynamics equation of the tethered systems is by means of the Lagrange's equations and various dynamic models of different tethered missions are established by the Lagrangian formulation. The Hamiltonian formulation is also widely used in the mechanical systems for its well-known symplectic structure property. With this in mind, the dynamics equation of the tethered system's motion are deduced by Hamilton's equations in this research. The relation between the Lagrangian and Hamiltonian is shown by Legendre transformation. The goodness of the Hamiltonian formulation is intuitive to reveal the Energy balance property that corresponds to the passivity property. Furthermore, the Hamiltonian energy function of tethered system is employed for facilitating the controller design. In order to bring the system into operations, the energy based control is to achieve the tethered system for precise positioning. Simulations are used to demonstrate the effectiveness of the designed controller.
\end{abstract}

Keywords-Tethered satellite system; Passivity; Energy-based control.

\section{INTRODUCTION}

Space tethered systems have been drawn great attention on its significant potential for the space missions utilization during the past decades. The tethered satellite system consists of two or more satellites which are connected by a long cable. The key challenge to bring the tethered space systems into operation is to successfully deploy the tether to the desired position in a stable and fast manner. However, the dynamics of the tethered systems are quite complex for the overall flexibility, then toward getting insight into the dynamics and control of the deployment for tethered systems, the simplified dumbbell model of the tethered systems are usually taken [1]. The mother and daughter satellites are considered as point mass connected by an inextensible massless tether. Thus, the important aspects of the dynamic behavior of the tethered system can be investigated by the simplified model without the involving the complicated mathematical equations.
In the past researches, the dynamics and control of tethered satellite system are well studied. The dynamic model of tethered system is usually derived by the Lagrangian formulation because it is easy to apply and solve the equations of motion for such two-body problem. A lot of research achievement of tethered systems are gained space applications such as the debris towed by tether, tethered satellite formation, tethered robot, etc. Among these applications, space tether deployment is the key issue, which is still a hot topic. The deployment control of the tethered system is not easy because it is prone to swing or liberating due to the coriolis force induced by deployment. Sometimes, the fast deployment speed may cause too large swing amplitude, which should be avoided to proceed space tethered operations. Many researchers are focusing on the problem how to achieve the tether deployment fast and stable with suppressing the libration [2-7]. For example, a tension control law in terms of the tether length and its velocity was firstly raised by Rupp for tether deployment [2]. Then, Fujii proposed a mission function control for the tether deployment based on the Lyapunov function [3]. A linear tension control for tether deployment was presented by Pradeep through the linearization [4]. Sun and Zhu developed a fractional order control law to eliminate the overshooting [5]. Further, Wen proposed a saturated controller by imposing a special saturation function to deal with tension constraints [6]. Kang and Zhu design a fractional order sliding mode tension control to counter the perturbations or uncertainties [7].

In this study, we proposed a natural controller design approach which is closely related to the dynamics of the tethered system. The energy-based control is used for precise positioning and effective suppressing the libration. Firstly, the dynamic equations are derived by the Lagrange's equations. The obtained dynamic equations of the tethered system are further represented in the compact form of Euler-Lagrange. The important passivity of the inherent aspect of tethered system's dynamics is revealed with specific matrix. In addition, Hamiltonian formulation is used to derive the dynamic equations of motion, which is an equivalent way of Lagrangian. Hamiltonian formulation is also widely used in the mechanical systems for its well-known symplectic structure property. Thus, energy balance property of the tethered system equivalent to passivity is given. The property is generally founded by 
Hamiltonian formulation rather than specifying the expressions of the matrix. Finally, the energy-based control is developed and asymptotic stability of the closed-loop system is proved to ensure the precise positioning.

\section{MODELING FORMULATION}

The widely used model of tethered space system control is the dumbbell model that is a long tether connecting two satellites at its ends, as shown in Fig.1 [8]. The system is moving in the orbit of the earth. The orbital motion coordinate is located as $o$-xyz. The $y$-axis directs toward the Earth center. The $x$-axis is perpendicular to the $y$-axis in the orbital plane and pointing at the velocity's direction of the orbit. The $z$-axis complies with the right-hand rule. The mass of the Main satellite and Subsatellite is $m_{1}$ and $m_{2}$, respectively. To bring insight of space tether system, following assumptions are made usually.

a) The field of the earth is central gravitational field and the Main and Sub satellites are particles.

b) The mass ratio of the Subsatellite over Main satellite is sufficiently small such that coordinate $o-x y z$ always remains in its nominal orbit.

c) The tether is massless and its flexibility is ignored.

d) The tether length $l$ is very short compared to the distance $R$ form coordinate $o-x y z$ to the center of earth.

e) External perturbation force is ignored. The orbit is circular and only motion in the orbital plane is considered.

f) Tether tension is the only control force acts along the tether. The tether is deployed/retrieved through the tension control, i.e., the required tension is produced by the motor driving the tether reel in/out system.

Based upon the assumptions, the TSS motion can be decomposed into the orbital motion of the system's center of mass (CM) and the local libration motion $\theta$ of tether about the $\mathrm{CM}$ in the orbital plane, as shown in Fig.1.

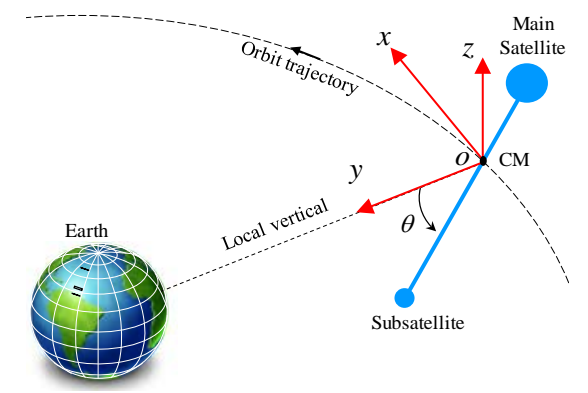

Figure 1. Sketch of the space tether system

Accordingly, the kinetic energy of the tethered system reads $K_{0}=0.5\left(m_{1}+m_{2}\right) R^{2} \Omega^{2}+0.5 m_{0} l^{2}\left(\theta^{\prime}+\Omega\right)^{2}+0.5 m_{0}\left(l^{\prime}\right)^{2}$, where the prime ( )' denotes the derivative with respect to time $t$, and $\Omega$ is the orbital angular velocity. $m_{0}=m_{1} m_{2} /\left(m_{1}+m_{2}\right)$ is the equivalent mass. Due to $l<R$, the tethered system's potential energy can be approximated $U_{0}=-\left(m_{1}+m_{2}\right) R^{2} \Omega^{2}+0.5 m_{0} l^{2} \Omega^{2}\left(1-3 \cos ^{2} \theta\right)$.

\section{A. Lagrangian Formulation}

Take the Lagrangian function as $L=K-U$, the dynamic equations of the tethered system can be straightly obtained according to the Lagrange's Equation,

$$
\frac{d}{d t} \frac{\partial L}{\partial \dot{s}}-\frac{\partial L}{\partial s}=Q
$$

where $s=\{l, \theta\}$ is the generalized coordinates and the nonconservative force $Q_{l}=-\bar{T}$ and $Q_{\theta}=0 . \bar{T}$ is the tether tension. Hence, the equations of motion are,

$$
\begin{aligned}
& l^{\prime \prime}-l\left[\left(\theta^{\prime}+\Omega\right)^{2}+\Omega^{2}\left(3 \cos ^{2} \theta-1\right)\right]=-\frac{\bar{T}}{m_{e}} \\
& l^{2} \theta^{\prime \prime}+2 l l^{\prime}\left(\Omega+\theta^{\prime}\right)+\frac{3}{2} l^{2} \Omega^{2} \sin 2 \theta=0
\end{aligned}
$$

For sake of simplicity, the dimensionless variables are introduced to normalize the equations of motion [6, 7], such as,

$$
\lambda=l / l_{n} \quad T=\bar{T} /\left(m_{0} l_{n} \Omega^{2}\right) \quad \tau=\Omega t \quad \dot{s}=\mathrm{d} s / \mathrm{d} \tau
$$

where $l_{n}$ is a nominal constant of tether length. $T$ is the dimensionless tension that is to regulate the tether deployment, $\tau$ is the dimensionless time which equals to the true anomaly. Then, the dimensionless model of tethered system is presented as,

$$
\begin{aligned}
& \ddot{\lambda}-\lambda\left[(1+\dot{\theta})^{2}-1+3 \cos ^{2} \theta\right]=-T \\
& \lambda^{2} \ddot{\theta}+2 \lambda \dot{\lambda}(1+\dot{\theta})+3 \lambda^{2} \sin \theta \cos \theta=0
\end{aligned}
$$

It should be noted here, $\lambda_{\max } \geq \lambda \geq \lambda_{\text {min }}>0$ to satisfy the physical real situation and avoid the singularity of second equation of the Eq.(3).

Further, to show the properties of tethered system, (3) is represented as the general form of Euler-Lagrange mechanical system,

$$
M(q) \ddot{q}+C(q, \dot{q}) \dot{q}+G(q)=u
$$

where $q=\operatorname{col}(\lambda, \theta)$ is the general coordinates, $M(q)$ is the mass matrix, $C(q, \dot{q})$ is the Coriolis and centrifugal force terms, $G(q)$ is the gravity term, and $u=\operatorname{col}(-T, 0)$ is the generalized force.

$$
M(q)=\left[\begin{array}{cc}
1 & 0 \\
0 & \lambda^{2}
\end{array}\right] C(q, \dot{q})=\left[\begin{array}{cc}
0 & -\lambda(\dot{\theta}+2) \\
\lambda(\dot{\theta}+2) & \lambda \dot{\lambda}
\end{array}\right] \quad G(q)=\left[\begin{array}{l}
-3 \lambda \cos ^{2} \theta \\
\frac{3}{2} \lambda^{2} \sin 2 \theta
\end{array}\right]
$$

From the dimensionless equations (4), a dimensionless Lagrangian $L(q, \dot{q})$ could be obtained as follows,

$$
L(q, \dot{q})=K(q, \dot{q})-U(q)=\frac{1}{2} \dot{\lambda}^{2}+\frac{1}{2} \lambda^{2}\left[(\dot{\theta}+1)^{2}-1+3 \cos ^{2} \theta\right]
$$


where $K(q, \dot{q})=\frac{1}{2} \dot{\lambda}^{2}+\frac{1}{2} \lambda^{2}\left[(\dot{\theta}+1)^{2}-1\right]$ and $U(q)=-\frac{3}{2} \lambda^{2} \cos ^{2} \theta$ are the dimensionless kinetic energy and potential energy, respectively.

Property 1. The inertia matrix $M(q)$ is positively bounded, $\zeta_{\min } \leq\|M(q)\| \leq \zeta_{\max } \cdot \zeta_{\min }$ and $\zeta_{\max }$ are the minimum and maximum eigenvalue of inertia matrix, respectively.

Property 2. There exists a positive constant $k_{g}$ satisfying $\|\partial G(q) / \partial q\| \leq k_{g}$.

Property 3. The time derivative of the mass matrix and the matrix of coriolis and centrifugal force terms are dependent with each other. Such that, $0.5 \dot{M}(q)-C(q, \dot{q})$ is a skewsymmetry matrix.

$$
\dot{q}^{T}[0.5 \dot{M}(q)-C(q, \dot{q})] \dot{q}=0
$$

Define an energy function $E$ satisfying the transformation $E=\dot{q}^{T} \frac{\partial L}{\partial \dot{q}}-L$, then one has,

$$
E=\frac{1}{2} \dot{\lambda}^{2}+\frac{1}{2} \lambda^{2} \dot{\theta}^{2}-\frac{3}{2} \lambda^{2} \cos ^{2} \theta=\frac{1}{2} \dot{q}^{T} M(q) \dot{q}+U(q)
$$

The derivative of the energy function can be directly obtained, such that,

$$
\begin{aligned}
\dot{E} & =\dot{q}^{T} M \ddot{q}+\frac{1}{2} \dot{q}^{T} \dot{M} \dot{q}+\dot{U} \\
& =\dot{q}^{T}\left(\frac{1}{2} \dot{M}-C\right) \dot{q}+\dot{q}^{T} u+\dot{q}^{T} G-\dot{q}^{T} G \\
& =\dot{q}^{T} u=-\dot{\lambda} T
\end{aligned}
$$

The energy function is $E$ bounded from below, if $\exists P \geq-\infty$, such that the potential energy function $U(q) \geq P$. Then, system satisfies passivity property as follows,

Property 4. Define the function $S=E-P \geq 0$ as the storage function, then the system (4) with inputs $u$ and outputs $\dot{q}$ is passive in general, such that,

$$
S(t)-S(0) \leq \int_{0}^{t} \dot{q}^{T} u \mathrm{dt}
$$

Assumption 1. The libration angle of the payload always remains in $(-\pi / 2, \pi / 2),|\theta|<\pi / 2$.

\section{B. Hamiltonian Formulation}

In Hamiltonian mechanics, the canonical coordinates $(q, p)$ is used to describe the physical system's motion. Related to the Lagrangian formulation, the Hamiltonian is a function of $(q, p)$ instead of $(q, \dot{q})$ in Lagrangian. It is understood that $\dot{q}$ is replaced by $q$ and $p$, thus $\dot{q}$ is in terms of $(q, p) \cdot p=\partial L / \partial \dot{q}$ is the generalized momentum.

The generalized momentum $p=\operatorname{col}\left(p_{\lambda}, p_{\theta}\right)$ is obtained as,

$$
\begin{gathered}
p_{\lambda}=\frac{\partial L}{\partial \dot{\lambda}}=\dot{\lambda} \\
p_{\theta}=\frac{\partial L}{\partial \dot{\theta}}=\lambda^{2}(\dot{\theta}+1)
\end{gathered}
$$

Invoking the Legendre transformation, the Hamiltonian function can be derived by,

$$
H(q, p)=\dot{q}^{T} p-L(q, \dot{q}(q, p))
$$

The Hamiltonian of the tethered satellite system is obtained, such that

$$
\begin{aligned}
H(q, p) & =\frac{1}{2} \dot{\lambda}^{2}+\frac{1}{2} \lambda^{2} \dot{\theta}^{2}-\frac{3}{2} \lambda^{2} \cos ^{2} \theta \\
& =\frac{1}{2} p_{\lambda}^{2}+\frac{\lambda^{2}}{2}\left(\frac{p_{\theta}}{\lambda^{2}}-1\right)^{2}+U(q)
\end{aligned}
$$

Hamilton Equations used for mechanical system is presented as follows,

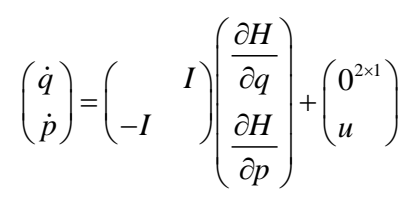

The motivation to use Hamiltonian formulation instead of Lagrangian formulation is the symplectic structure of Hamilton equations in (14). Then the equations of motion of the tethered system can be expressed as,

$$
\begin{aligned}
& \ddot{\lambda}=-\lambda \dot{\theta}^{2}+\lambda(\dot{\theta}+1)+3 \lambda \cos ^{2} \theta-T \\
& \lambda^{2} \ddot{\theta}+2 \lambda \dot{\lambda}(\dot{\theta}+1)=-3 \lambda^{2} \sin \theta \cos \theta
\end{aligned}
$$

It is easy to find that (15) is equivalent to (3) which is derived by the Lagrangian formulation.

Further, the time derivative of $H$ could be directly calculated as,

$$
\begin{aligned}
\dot{H}(q, p) & =\frac{\partial^{T} H}{\partial q} \dot{q}+\frac{\partial^{T} H}{\partial p} \dot{p} \\
& =\left(\frac{\partial^{T} H}{\partial q}, \frac{\partial^{T} H}{\partial p}\right)\left(\begin{array}{rr}
I & I
\end{array}\right)\left(\frac{\partial^{T} H}{\partial q}, \frac{\partial^{T} H}{\partial p}\right)^{T}+\frac{\partial^{T} H}{\partial p} u \\
& =-\frac{\partial H}{\partial p_{\lambda}} T=-\dot{\lambda} T
\end{aligned}
$$

Equation (16) indicates the energy balance property of tethered system. The increase in energy (Hamiltonian quantity) of the system equals to the power supply. The relation shown in (16) is actually equivalent with passivity (9), Moreover, it is quite straight to observe the intrinsic property of tethered system (3) by the Hamiltonian formulation.

\section{ENERGY-BASED CONTROL}

The aim of controller design is to deploy the tethered system to its desired position. As well known, the stable equilibrium position of the tethered system lies in local vertical. Thus, the desired states of tethered system is usually set at the 
downward equilibrium $(\lambda, \dot{\lambda}, \theta, \dot{\theta})=\left(\lambda_{d}, 0,0,0\right) . \lambda_{d}$ is the desired tether length and tether angle is zero. By virtue of the passive property of the tethered system, the controller design for tethered system deployment is posed based on the energy modified Lyapunov function in this section.

From the Hamiltonian formulation, the quantity of Hamiltonian energy function obtained by the Legendre transformation is lower bounded when the potential energy function $U(q)-P \geq 0$, then one can take a function $P=\bar{U}(q)=\left.U(q)\right|_{\theta=0}$ to shift the Hamiltonian function to be positive such that,

$$
V_{1}=H-P=\frac{1}{2} \dot{\lambda}^{2}+\frac{1}{2} \lambda^{2} \dot{\theta}^{2}-\frac{3}{2} \lambda^{2} \cos ^{2} \theta+\frac{3}{2} \lambda^{2}
$$

It is obvious that (17) is always positive. Thus, one can select the function $V_{1}$ as the Lyapunov function candidate, and then controller is designed to make the closed-loop system stable.

Take the derivative of the function $V_{1}$,

$$
\dot{V}_{1}=\dot{H}-\dot{P}=-T \dot{\lambda}+3 \lambda \dot{\lambda}
$$

Thus, the closed-loop tethered system is passive if the one take output $\dot{\lambda}$ and input $T$ as follows,

$$
T=3 \lambda+k_{v} \dot{\lambda}, k_{v}>0
$$

where $k_{v}$ is an strictly positive constant.

Then, substituting (19) into (18), which yields,

$$
\dot{V}_{1}=\dot{H}-\dot{P}=-T \dot{\lambda}+3 \lambda \dot{\lambda}=-k_{v} \dot{\lambda}^{2}
$$

It is straightforward to know that the closed-loop tethered system is Lyapunov stable under the controller (19). However, the final tether length is not certain since $V_{1}$ is not convex at $\lambda=\lambda_{d}, \theta=0$. It is easy to check with the Hessian Matrix of $V_{1}$, at the desired position, such that,

$$
\left.\frac{\partial^{2} V_{1}}{\partial q^{2}}\right|_{\substack{\lambda=\lambda_{d} \\
\theta=0}}=\left[\begin{array}{cc}
3 \sin ^{2} \theta & 3 \lambda \sin (2 \theta) \\
3 \lambda \sin (2 \theta) & 3 \lambda^{2} \cos (2 \theta)
\end{array}\right]_{\substack{\lambda=\lambda_{d} \\
\theta=0}}=\left[\begin{array}{cc}
0 & 0 \\
0 & 3 \lambda_{d}^{2}
\end{array}\right]
$$

It is obvious that the Hessian Matrix $\left.\frac{\partial^{2} V_{1}}{\partial q^{2}}\right|_{\substack{\lambda=\lambda_{d} \\ \theta=0}}$ is not positive definite. Thus, even if the tether may arrive to the desired length but it will not be able to stay there.

To achieve the desired final length along the vertical, the energy Lyapunov function should be modified so that it is convex at the desired position. For the purpose of this, we construct a Lyapunov function as follows,

$$
V_{2}=V_{1}+\frac{1}{2} k_{p}\left(\lambda-\lambda_{d}\right)^{2}
$$

where $k_{p}$ is an strictly positive constant. It is obvious to find that $V_{2} \geq 0$ and compared with $V_{1}$, a positive term $\frac{1}{2} k_{p}\left(\lambda-\lambda_{d}\right)$ is added, which has the unique minimum at desired tether length. Then, at the desired equilibrium we have,

$$
\begin{gathered}
\left.\frac{\partial V_{2}}{\partial q}\right|_{\substack{\lambda=\lambda_{d} \\
\theta=0}}=\left[\begin{array}{c}
3 \lambda \sin ^{2} \theta+k_{p}\left(\lambda-\lambda_{d}\right) \\
\frac{3}{2} \lambda^{2} \sin (2 \theta)
\end{array}\right]_{\substack{\lambda=\lambda_{d} \\
\theta=0}}=0 \\
\left.\frac{\partial^{2} V_{2}}{\partial q^{2}}\right|_{\substack{\lambda=\lambda_{d} \\
\theta=0}}=\left[\begin{array}{cc}
3 \sin ^{2} \theta+k_{p} & 3 \lambda \sin (2 \theta) \\
3 \lambda \sin (2 \theta) & 3 \lambda^{2} \cos (2 \theta)
\end{array}\right]_{\substack{\lambda=\lambda_{d} \\
\theta=0}}=\left[\begin{array}{cc}
k_{p} & 0 \\
0 & 3 \lambda_{d}^{2}
\end{array}\right]
\end{gathered}
$$

Thus, it indicates that $V_{2}$ is convex, then it is stable to stay at the desired equilibrium. Then, the controller (19) should be modified accordingly. The control law becomes,

$$
T=3 \lambda+k_{p}\left(\lambda-\lambda_{d}\right)+k_{v} \dot{\lambda}, k_{p}>0, k_{v}>0
$$

Theorem 1: Under the controller (25) and Assumption 1, the deployment control of the closed-loop tethered system is asymptotically stable at desired downward equilibrium.

Proof. Select the Lyapunov function candidate as $V_{2}$ and take the derivative of it, yields that,

$$
\dot{V}_{2}=-T \dot{\lambda}+3 \lambda \dot{\lambda}+k_{p}\left(\lambda-\lambda_{d}\right)
$$

Recall (25), one has,

$$
\dot{V}_{2}=-k_{v} \dot{\lambda}^{2} \leq 0
$$

From (27), the Lyapunov function $V_{2}$ is non-increasing, then one has $V_{2} \leq V_{2}(0)$. The trajectory of all the states will converge to the largest invariant set $W=\left\{q \in \mathbb{R} \mid \dot{V}_{2}=0\right\}$ as the time approaches to infinity. The set $W$ indicates $\dot{\lambda}=0$, which further means the tether length keeps constant $\lambda=$ constant . Then, recall (13) and (16), one can have,

$$
\frac{1}{2} \lambda^{2} \dot{\theta}^{2}-\frac{3}{2} \lambda^{2} \cos ^{2} \theta=\text { constant }
$$

Combine (28) with first equation of (15), we can know that there are at most two isolate solutions of $\dot{\theta}$ for $\dot{\theta} \in \mathbb{R}$. Then, one can have that,

$$
\ddot{\theta}=0
$$

Substitute (29) into the second equation of (15), yields,

$$
\sin \theta \cos \theta=0
$$

Further, the solutions of tether angle can be obtained under the Assumption 1, we have $\theta=0$. Combine with (25) and (15), we have,

$$
k_{p}\left(\lambda-\lambda_{d}\right)=0
$$

Then, one can get the unique solution for tether length $\lambda=\lambda_{d}$. Therefore, the deployment of tethered system has unique equilibrium at $(\lambda, \dot{\lambda}, \theta, \dot{\theta})=\left(\lambda_{d}, 0,0,0\right)$ under the controller (25) with the Assumption 1. The equilibrium 
$(\lambda, \dot{\lambda}, \theta, \dot{\theta})=\left(\lambda_{d}, 0,0,0\right)$ is concluded to be asymptotically stable, which completes the proof.

Remark 1: In the proof, the LaSalle's invariance theory is used to prove the asymptotic stability of the closed-loop system. However, it should be noted that it actually can't be directly applied because of unboundedness of tether angle. But it can be circumvented by variable transform as in [6, 10], which is omitted in the proof because it has no effect on the conclusion of asymptotic stability.

Remark 2: The convex of the function in (24) actually implies the asymptotically stable of the closed-loop system. Because the convex of the function at desired equilibrium means that any state of motion in the small domain near desired equilibrium has the minimum at desired equilibrium. That is the reason we construct the function to make the energy Lyapunov function have minimum at the desired equilibrium.

\section{SiMULTION AND DISCUSSION}

In this section, numerical experiments are carried on to verify the effectiveness of the proposed controller. The simulations are conducted in MATLAB R2017a using the numerical integrator ODE45. The tethered satellite system is assumed to orbit circularly with an altitude of $220 \mathrm{~km}$ and the orbital velocity is $1.18 \times 10^{-3} \mathrm{rad} / \mathrm{s}$. The maximum tether length is $100 \mathrm{~km}$. The corresponding initial and final parameters of the tether system are selected as $\left(\lambda_{0}, \dot{\lambda}_{0}, \theta_{0}, \dot{\theta}_{0}\right)=(0.01,0.5,0,0)$ and the desired states $\left(\lambda_{d}, \dot{\lambda}_{d}, \theta_{d}, \dot{\theta}_{d}\right)=(1,0,0,0)$, respectively.

Define two potential energy functions $U_{1}=U_{0}+P$ and $U_{2}=U_{1}+\frac{1}{2} k_{p}\left(\lambda-\lambda_{d}\right)^{2}$ according to the Lyapunov function $V_{1}$ and $V_{2}$. Parameters in these functions are set as $k_{p}=4$ and $\lambda_{d}=1$. Then, sketch the graph of the function in configuration space of $\lambda-\theta$, see Fig. 2. As shown in the graph of first subplot, the function $U_{1}$ has the minimum if the tether angle is zero but it is not depending on tether length. It indicates that the tethered system is able to deploy to the vertical direction but the deployed tether length is not certain maybe any value. However, seen from the second subplot, the function $U_{2}$ has the unique minimum at desired position. It can be observed that the function $U_{2}$ is convex around the small neighborhood of the desired equilibrium.

Further, the control laws (19) and (25) are used to clarify the stability of the closed-loop tethered system. The control gains are chosen as $k_{v}=1$ in (19) and $k_{p}=2, k_{v}=4$ in (25). The profiles of the position states and tension in tether are presented in Figs.3-5. Seen in Fig. 3, the length profiles show that both two proposed controller can achieve a stable tether deployment. The controller (25) fulfill the tether deployment to desired length successfully, however the controller (19) does not. It can be found that the tether angle profiles converge to zero as we want for both two controllers, see Fig. 4. The tension in tether for two controllers are shown in Fig.5, tension is positive and finally equal to the static balance force. In conclusion, the simulation results agree with the theoretical analysis given in Section III and the proposed controller (25) can perform the asymptotic stability for deployment control of the tethered system.
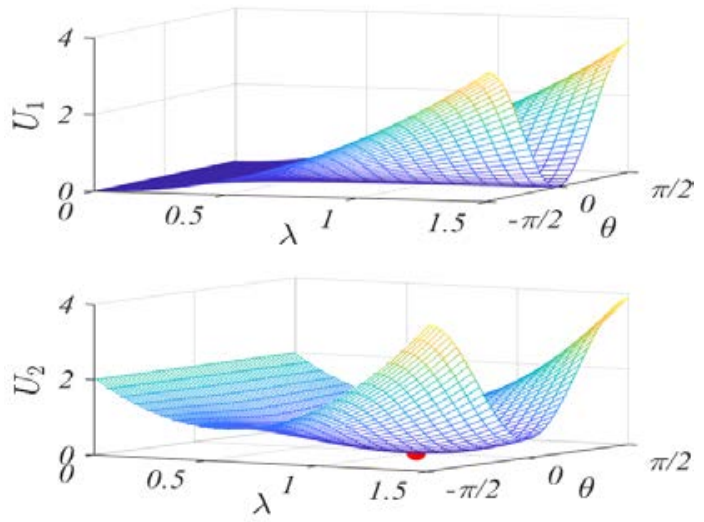

Figure 2. $U_{1}$ and $U_{2}$ in configuration space $\lambda-\theta$

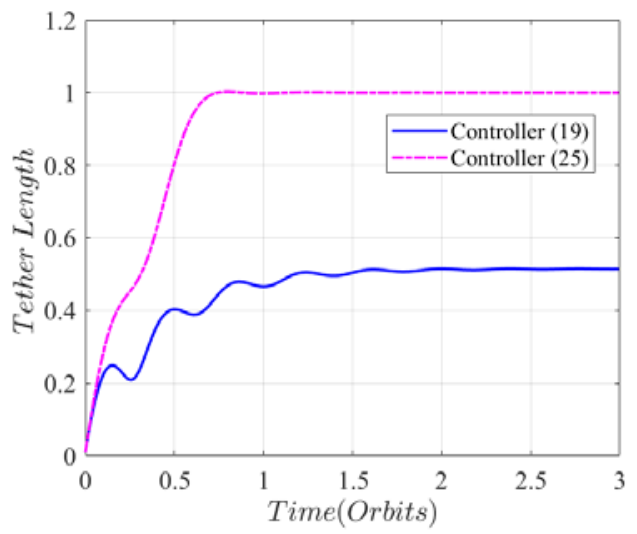

Figure 3. The deployed dimensionless tether length

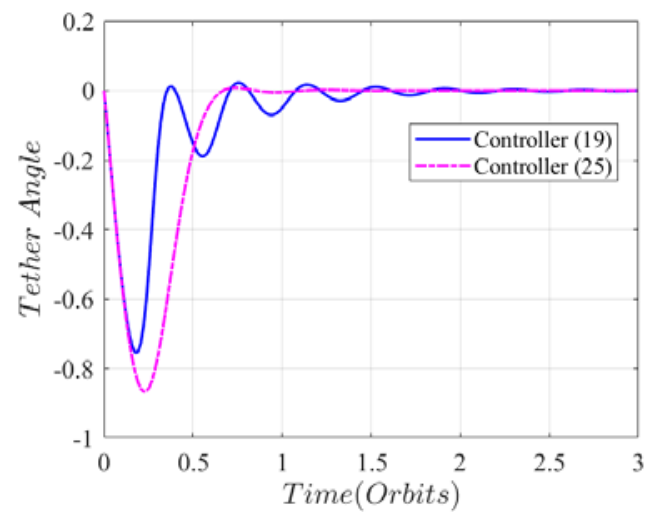

Figure 4. The tether angle during tether deployment 


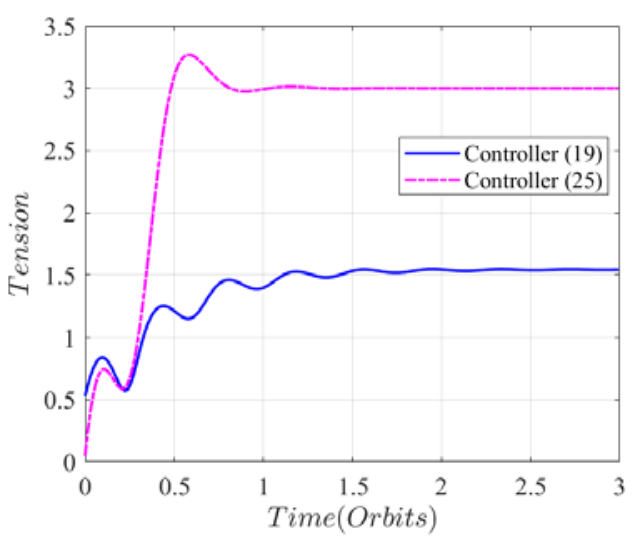

Figure 5. Tether tension during tether deployment

\section{CONCLUSTION}

In this study, we focus on the dynamics and control of space tethered system's deployment in two dimensional space. In order to get insight of the dynamic behavior of the tethered system, the dynamic model is formulated by the way of Lagrangian and Hamiltonian, respectively. In Lagrangian formulation, the dynamic equation is written into the EulerLagrange form and the passivity property is further presented by the specifically chosen matrix of coriolis/centrifugal force. In alternative way, the equivalent property of energy balance in the tethered system is performed by Hamiltonian formulation, which is much more intuitively due to the symplectic structure. This property is actually in general rather than depending on specified coriolis/centrifugal matrix. By virtue of the Hamilton formulation, the deployment control of the tethered system is studied based on Energy-based control methodology. Controllers are proposed for stabilizing tether deployment. The Lyapunov stability and asymptotical stability are analyzed for each controller, respectively. Finally, the results are verified by the numerical simulations.

\section{REFERENCES}

[1] K. D. Kumar, "Review on Dynamics and Control of Nonelectrodynamic Tethered Satellite Systems,” Journal of Spacecraft and Rockets, vol. 43, no. 4, pp. 705-720, July, 2006.

[2] C. C. Rupp, A tether tension control law for tethered subsatellites deployed along local vertical, Marshall Space Flight Center, Huntsville, AL, United States, September, 1975.

[3] H. Fujii, and S. Ishijima, "Mission function control for deployment and retrieval of a subsatellite," Journal of Guidance, Control, and Dynamics, vol. 12, no. 2, pp. 243-247, March, 1989.

[4] S. Pradeep, "A new tension control law for deployment of tethered satellites,” Mechanics Research Communications, vol. 24, no. 3, pp. 247-254, Feburary, 1997.

[5] G. Sun, and Z. H. Zhu, "Fractional-Order Tension Control Law for Deployment of Space Tether System,” Journal Of Guidance Control And Dynamics, vol. 37, no. 6, pp. 2062-2066, Nov, 2014.

[6] H. Wen, Z. H. Zhu, D. Jin, and H. Hu, "Space Tether Deployment Control with Explicit Tension Constraint and Saturation Function," Journal of Guidance, Control, and Dynamics, vol. 39, no. 4, pp. 916-921, April, 2015.

[7] J. Kang, Z. H. Zhu, W. Wang, A. Li, and C. Wang, "Fractional order sliding mode control for tethered satellite deployment with disturbances,” Advances in Space Research, vol. 59, no. 1, pp. 263-273, January, 2017.

[8] M. P. Cartmell, and D. J. McKenzie, "A review of space tether research,” Progress in Aerospace Sciences, vol. 44, no. 1, pp. 1-21, January, 2008.

[9] J. Kang, Z. H. Zhu, W. Wang, C. Wang, and A. Li, "Energy-based output feedback tension control for space tether deployment under physical constraints”, in 2018 American Control Conference, 2018 (in press).

[10] R. Lozano, I. Fantoni, and D. J. Block, "Stabilization of the inverted pendulum around its homoclinic orbit," Systems \& Control Letters, vol. 40, no. 3, pp. 197-204, 2000. 